\title{
Assistência à mulher climatérica: novos paradigmas
}

\author{
Assistance to the climacteric woman: new paradigms \\ Atención a la mujer climatérica: nuevos paradigmas
}

\section{Dino Roberto Soares De Lorenzi', Lenita Binelli Catan', Karen Moreira", Graziela Rech Ártico'}

'Universidade de Caxias do Sul. Departamento de Tocoginecologia. Caxias do Sul, RS

"Universidade de Caxias do Sul. Ambulatório Multidisciplinar de Atenção ao Climatério. Caxias do Sul, RS

Submissão: $30 / 06 / 2008$

Aprovação: 03/02/2009

\section{RESUMO}

O envelhecimento populacional é uma realidade demográfica brasileira. Como conseQüência, espera-se, nos próximos anos, um aumento progressivo na procura dos serviços de saúde por mulheres com Queixas relacionadas ao climatério. Paralelamente, a assistência ao climatério tem passado por uma modificação de paradigmas, impondo aos profissionais de saúde uma mudança de atitude. Reconhecese que o climatério é influenciado tanto por fatores biológicos, como por fatores psicossociais e culturais, cujo conhecimento é fundamental para uma assistência mais Qualificada e humanizada. Este artigo propõe refletir sobre as mudanças de paradigmas na assistência ao climatério, destacando a multidisciplinaridade e interdisciplinaridade, no sentido acolher melhor essa parcela da população e proporcionarIhe um cuidado integral e individualizado, aproximando o saber da sensibilidade, voltado a uma melhor Qualidade vida.

Descritores: Climatério; Menopausa; Qualidade de vida; Saúde da mulher.

\section{ABSTRACT}

Population aging is a demographic reality for Brazil. ConseQuently, in the next years it is expected a progressive increase in seeking health care services in the country by women with complaints related to climacterium. Parallel to it, assistance at this part of woman's life has been going through a paradigm shift which has imposed to health professionals a change of attitude in relation to this stage of woman's life. Today it is acknowledged that the climacterium is influenced by biological, psychosocial and cultural factors, whose knowledge is fundamental for planning a more eualified and humanized care. This article proposes a reflection on the paradigm shifts in assistance at climacterium, highlighting important aspects as multidisciplinarity and interdisciplinarity, so as to serve better this portion of population, and provide it with more integrated and individualized care, bringing together knowledge and sensitivity, and always aiming at a better Quality of life.

Descriptors: Climacteric; Menopause; Quality of life; Women's health.

\section{RESUMEN}

Envejecimiento poblacional es una realidad demográfica brazilian. Consecuentementese espera un aumento progresivo de la búsqueda en los servicios de salud del país por mujeres con Quejas relacionadas al climaterio. La asistencia al climaterio ha pasado por un cambio de paradigmas que han impuesto a los profesionales de la salud. El climaterio es influenciado por factores biológicos, factores psicosociales y culturales, cuyo conocimiento es fundamental para la planificación de una asistencia. Artículo se propone reflexionar sobre los cambios de paradigmas, el abordaje multidisciplinario e interdisciplinario, en el sentido de recibir mejor esta parcela de la población y proporcionarle un cuidado integral e individualizado, aproximando el saber de la sensibilidad, y el tener como objetivo a una mejor calidad de vida.

Descriptores: Climaterio; Menopausia; Calidad de vida; Salud de la mujer. 


\section{INTRODUÇÃO}

A partir dos anos 80, o envelhecimento populacional tornou-se um fenômeno global, inicialmente observado nos países desenvolvidos e, mais recentemente, nos países em desenvolvimento ${ }^{(1)}$. Segundo projeções da Organização Mundial da Saúde, entre 1990 e 2025, o número de idosos aumentará entre sete e oito vezes em vários países da América Latina, África e Ásia ${ }^{(2)}$.

O Brasil, à semelhança de outros países latino-americanos, passa por um processo de envelhecimento populacional acelerado e intenso. Entre 1980 e 2000, a proporção de brasileiros com mais de 60 anos aumentou de $6,1 \%$ para $8,6 \%$, devendo chegar a $14 \%$ até 2025 , o Que representará uma das maiores populações de idosos no mundo ${ }^{(1,3,4)}$.

As implicações sociais e concernentes ao envelhecimento populacional no âmbito da saúde pública são preocupantes, pois, ao contrário dos países mais desenvolvidos, onde o aumento da expectativa de vida foi gradual e aliado a um maior desenvolvimento tecnológico e científico, o que possibilitou proporcionar uma maior Qualidade de vida e saúde a essa parcela da população, o mesmo não ocorreu nos países em desenvolvimento ${ }^{(2,4,5)}$.

No Brasil, a atenção à saúde da população mais idosa ainda é partilhada com Questões não totalmente resolvidas, como a mortalidade infantil e o controle das endemias, o que divide não somente os profissionais de saúde, mas também os recursos financeiros disponíveis para investimentos em saúde. Nesse contexto, não se pode desconsiderar a maior ocorrência de afecções crônicodegenerativas entre essa parcela da população, em particular Quando o envelhecimento populacional não é acompanhado de políticas de saúde específicas voltadas à promoção e prevenção em saúde ${ }^{(2)}$.

\section{RESULTADOS E DISCUSSÃO}

\section{Envelhecimento Feminino: o Desafio do Climatério}

A despeito de referências acerca do climatério possam ser encontradas em textos escritos por Aristóteles (384-322 a.C.), até a poucas décadas, a condição de mulher "menopausada" era raramente expressa em público, devido ao constrangimento Que isso causava, fazendo com Que pouco se conhecesse acerca das suas necessidades e demandas. Em parte, a pouca atenção prestada ao climatério no passado deveu-se à menor expectativa de vida feminina até então. Poucas mulheres viviam o suficiente para chegar ao climatério, situação Que mudou com o progressivo aumento da expectativa de vida feminina a partir da segunda metade do século $\mathrm{XX}^{(4-7)}$

Segundo Rosembaum ${ }^{(8)}$, atualmente, várias são as razões pelas Quais o período do climatério tem merecido uma maior atenção no âmbito da saúde pública. Inicialmente, o aumento do número de mulheres com mais de 50 anos na população mundial, Que de 467 milhões em 1990 deverá chegar a 1,2 bilhões em 2030. Além disso, é necessário considerar a crescente participação dessas mulheres no mercado de trabalho e geração de divisas.

No Brasil, o envelhecimento populacional mostra uma clara tendência à feminização. As mulheres com mais de 40 anos correspondem a $32 \%$ da população feminina, percentual este Que deve aumentar em $11 \%$ até 2010 . Além disso, a sua expectativa de vida já ultrapassa em 10,8 anos à masculina, chegando aos 75,6 anos, fazendo com Que ocorra uma maior procura nos serviços de saúde brasileiros por mulheres com Queixas relacionadas ao climatério $^{(7,9)}$.

Frente a esta nova realidade demográfica e as novas orientações na atenção clínica a essas mulheres, advindas de recentes pesQuisas, a assistência ao climatério tem passado por uma intensa mudança de paradigmas em busca de uma assistência mais integral e humanizada. ConseQüentemente, o conhecimento das condições de saúde dessa parcela da população, suas demandas por serviços médicos e necessidades sociais tornou-se mais prioritário do Que nunca para a formulação de políticas de saúde voltadas a um envelhecimento feminino mais sadio, menos oneroso e com mais Qualidade de vida ${ }^{(5)}$.

\section{Climatério: Definição e Aspectos Clínicos}

O climatério é um fenômeno endócrino decorrente do esgotamento dos folículos ovarianos Que ocorre em todas as mulheres de meia idade. Inicia-se entre os 35 e 40 anos, estendendo-se aos 65 anos, caracterizando-se por um estado de hipoestrogenismo progressivo ${ }^{(10-13)}$.

O esgotamento folicular inicia-se ainda na vida intra-uterina, na $22^{\mathrm{a}}$ semana de gestação, o ovário possui entre seis e oito milhões de oócitos primários Que, por meio de um processo contínuo de atresia, reduzem-se a dois milhões no nascimento e a $300.000 \mathrm{ou}$ 400.000 na menarca. O processo de atresia continua-se a cada ciclo menstrual até o total esgotamento folicular, levando a uma Queda progressiva da secreção de estradiol, com manifestações sistêmicas. A suspensão definitiva dos ciclos menstruais ou menopausa reflete a ausência de níveis de estradiol suficientes para proliferar o endométrio ${ }^{(10-12)}$.

Aproximadamente $50 \%$ a $70 \%$ das mulheres referem sintomas somáticos e dificuldades emocionais nos anos Que seguem a menopausa, com destaque para ondas de calor ou fogachos, devido as suas implicações negativas para a sua Qualidade de vida ${ }^{(10-12)}$.

O mecanismo fisiopatológico das ondas de calor ainda não é totalmente conhecido, porém, sabe-se Que o declínio dos níveis de estradiol interfere no centro regulador da temperatura localizado no hipotálamo, favorecendo a sua ocorrência. Caracterizam-se por uma sensação transitória e súbita de aumento da temperatura corporal, freqüentemente acompanhada de sudorese, palpitações e cefaléia, Que acaba por interferir nas atividades diárias e na Qualidade do sono ${ }^{(10-12)}$.

A atrofia urogenital é outra Queixa comum nessa fase e Que pode causar intenso desconforto à mulher. A intensificação do declínio estrogênico após a menopausa promove um adelgaçamento e enrijecimento da parede vaginal, Que aliados a uma menor lubrificação, causam dispareunia e dificuldades no intercurso sexual. O terço distal da uretra sofre também um processo de atrofia, Que se manifesta por disúria e urgência miccional ${ }^{(1,12)}$.

Na esfera cognitivo-comportamental, no climatério, não são raras mudanças comportamentais, maior labilidade emocional e até dificuldades com a memória. Não obstante, há dúvidas acerca do Quanto tais Queixas estariam relacionadas à Queda estrogênica ou a fatores psicossociais e orgânicos relacionados ao processo de envelhecimento. $\mathrm{O}$ hipoestrogenismo interferiria na síntese dos neurotransmissores, modulando o comportamento e sintomas psíquicos. As próprias ondas de calor, dependendo da sua 
intensidade e freqüência, por interferirem no sono e nas atividades cotidianas, seriam causa de irritabilidade e até depressão. Para outros autores, a maior ocorrência de depressão no climatério estaria relacionada principalmente ao medo de envelhecer aliado a sentimentos de inutilidade e carência afetiva ${ }^{(12,13)}$.

Não se pode desconsiderar também Que, nas culturas ocidentais, a beleza física, a juventude e a maternidade são elementos de valorização da mulher, cuja perda pode favorecer sentimentos de desvalia, tristeza e até depressão. A maior percepção do envelhecimento e a intensificação da sintomatologia decorrente do hipoestrogenismo, aliados a eventos comuns nessa fase, como o crescimento dos filhos, a aposentadoria ou a morte do cônjuge demandam ajustes emocionais e mudanças de estilo de vida por vezes difíceis para a mulher ${ }^{(5,12-14)}$. Ao mesmo tempo, cabe lembrar Que, no climatério, as mulheres tendem a atribuir indiscriminadamente à menopausa tanto os sintomas relacionados à carência estrogênica, como as Queixas físicas ou emocionais decorrentes de eventuais estados mórbidos prévios, confundindo-as ${ }^{(15)}$.

A sexualidade merece particular atenção no climatério. Esta é reconhecida como um dos pilares da Qualidade de vida, porém a sua abordagem nem sempre é feita adequadamente no climatério por constrangimento das mulheres ou despreparo dos próprios profissionais de saúde em lidar com essa Questão. Além disso, conforme lembrado por Favarato (2000), a sexualidade teria um caráter multidimensional, sendo influenciada por fatores psicossociais e culturais, em especial por relacionamentos interpessoais e experiências de vida, não se restringindo a fatores anatômicos e hormonais ${ }^{(16)}$.

Entre $25 \%$ e $33 \%$ das mulheres entre 35 e 59 anos apresentam disfunções sexuais, podendo chegar a 75\% entre os 60 e 65 anos. Além das dificuldades decorrentes da atrofia urogenital, a mulher climatérica tem de lidar com as mudanças físicas decorrentes do envelhecimento simbolizadas pela menopausa, Que podem comprometer a percepção da sua auto-imagem corporal, diminuindo a sua auto-estima e interferindo negativamente na sua libido. Esse processo diverge de mulher para mulher, pois, entre as Que vivenciam mais positivamente o climatério, as repercussões na esfera sexual parecem ser menos intensas. Estas perceberiam na redução das obrigações com os filhos e com a carreira profissional, uma oportunidade para o exercício afetivo-sexual ${ }^{(17,18)}$.

Ainda em relação à esfera sexual, é preciso lembrar Que os homens pouco conhecem acerca do climatério e as suas implicações para a saúde da mulher, tendo por vezes dificuldade de compreender o processo por Que esta está passando. Agravando a situação, após os 50 anos, o homem sofre um declínio natural dos níveis de testosterona, o Que pode comprometer sua libido e potência sexual. Ao mesmo tempo, após os 60 anos, a ocorrência de neoplasias de próstata é maior, podendo o seu tratamento interferir na atividade sexual ${ }^{(5,16-18)}$

Dificuldades com o sono são também comuns em mulheres após os 50 anos, em especial a insônia, ainda Que, até o momento, não tenha sido possível estabelecer uma associação definitiva destas com a Queda estrogênica, sendo atribuídas, na maioria das vezes, à ocorrência de fogachos ou a dificuldades emocionais. Sabe-se que o despertar acompanhado de fogachos despende mais energia, resultando em fadiga, irritabilidade e até depressão(19).

O ganho ponderal excessivo é freQüentemente relatado pelas mulheres após a menopausa, o Que merece atenção não apenas pela Questão estética, mas sim pelas implicações no risco cardiovascular e na gênese do câncer de mama, endométrio e cólon. No climatério, o ganho ponderal chega a $0,8 \mathrm{Kg} / \mathrm{ano}$; porém, após a menopausa, pode haver um aumento de $20 \%$ na gordura corporal. O papel do hipoestrogenismo nesse processo é ainda incerto, Que parece estar mais relacionado à inadeQuação da dieta às necessidades energéticas da mulher climatérica. A carência estrogênica contribuiria principalmente para o acúmulo de gordura abdominal, aumentando o risco cardiovascular feminino ${ }^{(20,21)}$

Das co-morbidades associadas ao hipoestrogenismo climatérico, merece destaque a osteoporose e a maior incidência de doenças cardiovasculares. Após a menopausa, a velocidade de perda óssea pode chegar a 2\% ao ano. Nos Estados Unidos, a osteoporose é responsável anualmente por 1,2 milhões de fraturas, com taxas de mortalidade entre $12 \%$ e $20 \%$. As mulheres sobreviventes desenvolvem seqüelas muitas vezes permanentes e que requerem medidas de reabilitação extremamente dispendiosas ${ }^{(22)}$.

No Que se refere às doenças cardiovasculares, durante o menacne, a incidência de cardiopatia işuêmica feminina é cerca de três vezes menor Que a masculina. Após a menopausa, com a Queda dos níveis de estradiol, o risco cardiovascular feminino aumenta progressivamente, equivalendo-se ao do homem aos 75 anos $^{(23)}$. Para se ter uma idéia da importância dessa Questão, vale lembrar Que, no Brasil, no ano de 2000, a doença cardiovascular isQuêmica levou ao óbito 32.936 mulheres, eneuanto Que o câncer de mama foi responsável por 8.308 óbitos $^{(5)}$.

\section{A Assistência à Mulher Climatérica: a Busca por Novos Paradigmas}

Até recentemente, a assistência à mulher climatérica centravase principalmente na terapia hormonal. Entretanto, a partir de 2002, a publicação dos resultados dos estudos conhecidos por WHI (Women's Health Initiative), houve uma sensível redução na sua prescrição $^{(5,24)}$.

Os achados do estudo WHI apontaram para um risco significativo maior para eventos tromboembólicos e câncer de mama, respectivamente, no final do primeiro e após o Quinto ano do uso contínuo de estrógenos conjugados eqüinos associados a acetato de medroxiprogesterona, Quando comparado com um grupo controle sem QualQuer medicação hormonal. Ainda Que, posteriormente, tais resultados tenham sido Questionados por problemas metodológicos, acabaram por levar a uma maior reflexão acerca da assistência à mulher climatérica ${ }^{(5,22,24)}$.

As indicações da terapia hormonal tornaram-se restritas ao alívio dos sintomas vasomotores e da atrofia urogenital e na prevenção da osteoporose, não tendo sido esta última indicação referenda pelo FDA norte-americano ${ }^{(25)}$. Tal conservadorismo em relação à prescrição à terapia hormonal tem sido contestado por pesquisas recentes, cujos achados têm apontado para possíveis efeitos benéficos desta. Dentre estas, uma revisão do próprio estudo WHI demonstrou Que a terapia hormonal teria um efeito de proteção cardiovascular Quando iniciada nos primeiros dez anos após a menopausa, Quando o processo de aterosclerose ainda é inicial ${ }^{(26)}$. No entanto, a despeito destes estudos, até o momento a prescrição ampla de estrógenos no climatério permanece não recomendada ${ }^{(25)}$.

Para as mulheres, a mudança de comportamento em relação à 
prescrição da terapia hormonal tem sido difícil de compreender, gerando indagações e até desconfianças acerca dos seus motivos. Até o estudo $\mathrm{WHI}$, a terapia hormonal representava uma resposta aos anseios das mulheres em promover a sua saúde nos anos Que se seguiam a menopausa. Vale lembrar Que nessa fase, são muitas as fantasias e dúvidas femininas em relação às implicações da menopausa para a saúde. Em parte, isso se deveu à excessiva pressão da indústria farmacêutica na década passada, Que estimulava o uso praticamente irrestrito da terapia hormonal, bem como da inadequada e excessiva exploração do climatério pelos meios de comunicação, situação essa agravada pela dificuldade dos médicos em lidar com a complexidade da síndrome climatérica, restringindoa freeüentemente a intervenções medicamentosas ${ }^{(5,11)}$.

O consenso atual é que a Qualidade de vida seja o norteador de Qualeuer intervenção no climatério ${ }^{(5)}$. Em parte, a preocupação atual com os aspectos relacionados à Qualidade de vida ao longo do ciclo vital humano originou-se do reconhecimento pelos profissionais de saúde da importância dos sentimentos e percepções dos indivíduos, bem como da monitorização do seu bem estar frente a medidas terapêuticas tomadas com vistas a prolongar a vida, aliviar a dor, restaurar funções ou prevenir incapacidades ${ }^{(27)}$.

Para a Organização Mundial da Saúde, Qualidade de vida referese à percepção Que um indivíduo tem de sua vida no sistema de valores e na cultura em Que vive, com base em suas metas e expectativas $^{(5.9)}$. Os fatores físicos, mentais, psicológicos, sociais, econômicos, culturais e espirituais, exercem influência na saúde física na Qualidade de vida. A qualidade de vida é definida como um construto multidimensional, com significados diferenciados segundo a diversidade de contextos de vida. Entre as dimensões Que lhe dão significado, estão a manutenção da capacidade funcional, a satisfação pessoal, o estado emocional e a interação social ${ }^{(5,9,18)}$.

Dentre os fatores associados à Qualidade de vida da mulher no climatério, os mais relevantes são as suas condições físicas e emocionais prévias, bem como a sua inserção social e experiências frente a eventos vitais. Mais recentemente, tem sido reconhecida a influência das atitudes e percepções da mulher em relação à menopausa na Qualidade de vida no climatério ${ }^{(12,15,18)}$.

Mulheres com uma percepção mais negativa da menopausa não somente tendem a apresentar uma pior Qualidade de vida, como sintomas climatéricos mais severos ${ }^{(12,27)}$. As ondas de calor parecem ser os únicos sintomas diretamente associados com a Queda estrogênica, ao passo que as demais Queixas climatéricas (labilidade emocional, menor libido e insônia) estariam relacionadas principalmente ao modo como a menopausa é percebida ${ }^{(12)}$.

Frente ao exposto acima, é reconhecida a necessidade de se buscar um paradigma mais abrangente, não somente explicativo, mas sim interpretativo das Questões relacionadas à saúde da mulher climatérica. A percepção atual é de Que o fenômeno saúde em todas as fases da vida esteja conectado a uma realidade social específica, sendo influenciado por fatores políticos, econômicos e culturais, não se restringindo a fatores biológicos. O desafio está em não reduzir a assistência médica a intervenções meramente curativas, visto Que os indivíduos não são totalmente saudáveis ou doentes, mas apresentam diferentes níveis de saúde e de doença, conforme o seu momento de vida ${ }^{(5,18,28)}$.

A assistência ao climatério está se expandindo para além dos aspectos biológicos relacionados ao hipoestrogenismo, passando a considerar também a influência de fatores culturais e psicossociais, na busca de um cuidado mais integral e efetivo(11). Para tanto, é necessário Que os profissionais de saúde, médicos ou não, procurem perceber a mulher climatérica na sua integralidade, individualizando as suas necessidades e disponibilizando tanto medidas de promoção à saúde, como terapêuticas e de reabilitação com vistas a proporcionar-lhe uma melhor Qualidade de vida. Com isso se evitará a regulação indevida do corpo da mulher e a "medicalização" do envelhecimento feminino, abrindo um espaço para a participação da própria mulher nas decisões sobre o seu planejamento terapêutico ${ }^{(5,29-31)}$.

Sem desconsiderar o papel da terapia hormonal no alívio das ondas de calor e dos sintomas urogenitais, têm sido resgatadas medidas igualmente importantes na promoção da saúde e Qualidade de vida no climatério, como o cuidado alimentar, o combate ao sedentarismo e a restrição ao tabagismo. A educação em saúde, em especial, tem merecido destaque, não somente por contribuírem para um maior autocuidado, como para a mudança de eventuais posicionamentos negativos acerca do envelhecimento feminino, esclarecendo dúvidas acerca do climatério e da menopausa e, conseqüentemente, reduzindo a ansiedade da mulher ${ }^{(5,18)}$. Estudo examinou o impacto das intervenções educativas em um grupo de mulheres climatéricas residentes em Londres, tendo mostrado Que, após 15 meses, as primeiras não somente passaram a expressar menos crenças negativas acerca do climatério, como, inclusive, menos sintomas ${ }^{(32)}$.

O sedentarismo, por sua vez, favorece a perda da massa muscular, a redução da taxa metabólica de repouso e da capacidade aeróbica, bem como uma maior resistência insulínica e o acúmulo de adiposidade. Estudos demonstraram Que mulheres climatéricas com atividade física regular tendem a manifestar menos sintomas somáticos e uma melhora do humor, bem como um alívio nas ondas de calor ${ }^{133-}$ 35). O exercício físico atuaria estimulando a secreção de â-endorfinas hipotalâmicas, substâncias Que promovem a sensação de bem estar e contribuem para a estabilização da termorregulação hipotalâmica( ${ }^{(32)}$.

A atividade física regular contribui também para a preservação da mobilidade articular e o fortalecimento muscular, melhorando a coordenação motora. Além disso, aumenta a densidade mineral óssea, melhora a capacidade respiratória e o perfil lipídico, diminui a freeüência cardíaca de repouso e a gordura corporal, além de normalizar a pressão arterial, contribuindo assim para uma menor incidência de co-morbidades ósseas e cardiovasculares ${ }^{(33,34)}$. Ademais, melhora a imagem corporal da mulher, aumentando a sua auto-estima e a disponibilidade para o exercício afetivo-sexual ${ }^{(5)}$.

O tabagismo, em especial, tem se mostrado extremamente nocivo à mulher climatérica, contribuindo para uma maior deterioração da sua Qualidade de vida, devendo ser combatido nessa fase. Os seus malefícios incluem a doença bronco-pulmonar obstrutiva crônica, o câncer de pulmão e o maior risco cardiovascular. A nicotina estimula ainda a secreção de serotina e de dopamina, causando ansiedade e até euforia, além de interferir na globulina carreadora de estrogênio, agravando os sintomas climatéricos e o risco de osteoporose $^{(36,37)}$.

Dentre os agravos à saúde mais prevalentes no climatério, grande parte relaciona-se direta ou indiretamente à ingestão inadequada de alimentos, Quer seja em excesso ou deficiência por longos períodos $^{(20,38)}$. O consumo em excesso de alimentos energéticos, 
principalmente gorduras saturadas, associa-se a um maior risco cardiovascular. Em contrapartida, a baixa ingestão de nutrientes, como o cálcio, favorece a ocorrência de osteoporose. Estudo envolvendo 154 mulheres climatéricas residentes na cidade de São Paulo revelou Que 75\% apresentavam sobrepeso ou obesidade e deficiências importantes de vitaminas, ferro e cálcio ${ }^{(38)}$. Achados similares foram observados em levantamento realizado no Ambulatório de Climatério da Universidade de Caxias do Sul ${ }^{(20)}$.

Assim, a despeito das várias são as possibilidades de intervenção no climatério, hoje se reconhece Que para serem efetivas, devem ser sempre precedidas de uma escuta Qualificada, Que permita identificar as reais necessidades da mulher nessa fase. É preciso Que esta tenha espaço para manifestar a sua percepção e sentimentos acerca do momento Que está vivenciando e as suas dificuldades pessoais, devendo ser informada sobre as mudanças Que o seu corpo está sofrendo e as implicações para a sua saúde. O climatério precisa ser entendido como um período normal de transição, em Que a prevenção de doenças e o alívio de possíveis desconfortos podem ser abordados de diferentes maneiras ${ }^{(5,30)}$.

Infelizmente, no Brasil, a mulher climatérica nem sempre encontra o acolhimento necessário ao atendimento de suas necessidades. A assistência médica é geralmente fragmentada, propondo intervenções meramente curativas, ainda Que o climatério não seja um estado mórbido, mas uma etapa normal do envelhecimento feminino $^{(5,18,30)}$.

É preciso Que os profissionais de saúde busquem o que está oculto por trás da Queixa referida, Quais os seus anseios e necessidades não explicitados pela mulher climatérica Que os procura, como esta vive e Quais as suas expectativas nos anos Que se seguem a menopausa ${ }^{(5,39)}$. Infelizmente, tal prática se encontra ainda distante do cotidiano da maioria dos serviços de saúde brasileiros, sejam estes públicos ou privados. O climatério persiste sendo percebido como uma entidade patológica, Que demanda basicamente intervenções medicamentosas ${ }^{(5)}$.

Para contornar essa situação, é premente Que a mulher climatérica passe a ser percebida na sua integralidade, de forma Que, além de ser ouvida nas suas Queixas, tenha acesso, de forma particularizada e individualizada, tanto a medidas de promoção e prevenção em saúde, como terapêuticas e de reabilitação, com vistas a uma melhor Qualidade de vida ${ }^{(5,40,41)}$.

A questão da individualização e da participação da própria mulher nas decisões relativas à assistência médica durante o climatério é fundamental nessa era pós-WHI. Os próprios consensos atuais sobre a prescrição da terapia hormonal ressaltam a importância da participação da mulher no seu plano terapêutico, sempre à luz das evidências científicas. Tal medida é essencial para Que se fuja da tendência à regulação excessiva do corpo da mulher climatérica através da "medicalização" indevida do envelhecimento feminino ${ }^{(5,41)}$.

No campo da saúde, a integralidade compreenderia um agrupamento de tendências cognitivas e políticas imbricadas entre si, ainda Que não completamente articuladas, visto a dificuldade de atender plenamente a totalidade das demandas do ser humano. No enfoque da integralidade, a abordagem das Questões de saúde compreende tanto dimensões assistenciais, Quanto preventivas, contemplando assim tanto os portadores de alguma doença ou agravo, como os indivíduos considerados saudáveis. Tal premissa se aplica claramente à mulher climatérica ${ }^{(40)}$.

Quando preocupados com a Questão da integralidade, os profissionais de saúde comprometem-se em buscar o verdadeiro significado das Queixas climatéricas, de forma a se atender a mulher e não o problema referido. Ao mesmo tempo, o momento da consulta não deixa de ser otimizado para identificar possíveis agravos não manifestos ou situações de risco. Nesse contexto, a intervenção de caráter multidisciplinar e interdisciplinar ganha espaço por potencializar e Qualificar a intervenção médica ${ }^{(39)}$.

A integralidade representa, talvez, o maior desafio nas práticas clínicas e político-sanitárias atuais, pois demanda um rompimento no processo tradicional e até cristalizado na forma como são feitas as intervenções em saúde. Não se Quer aQui desconsiderar a importância da ação médica especializada, porém, dependendo da forma como ela é conduzida, pode favorecer a própria iatrogenia, conforme observado em relação à terapia hormonal na década $\operatorname{passada}^{(39)}$.

\section{CONSIDERAÇÕES FINAIS}

Indubitavelmente, várias são as possibilidades de intervenção no climatério, cuja efetividade depende de uma escuta Qualificada dessas mulheres, das Questões ocultas em suas Queixas, dos seus sentimentos e percepções acerca do seu envelhecimento. Para tanto, é indispensável Que a mulher climatérica tenha espaço para expressar os seus sentimentos acerca do momento Que está vivendo e as dificuldades Que está sentindo, recebendo informações sobre as mudanças Que o seu corpo está sofrendo e as suas implicações para a sua saúde. Ao mesmo tempo, não se pode mais restringir a saúde a Questões meramente orgânicas.

No climatério, em especial, é preciso rever a subjetividade da mulher, resgatando a sua história pessoal, valores, expectativas e desejos, evitando abordagens mecanicistas e reducionistas, através de uma prática Que aproxime o saber da sensibilidade. Ainda Que o envelhecimento seja uma conQuista das nações civilizadas e progressistas, cabe lembrar Que não basta apenas maximizar a expectativa de vida, mas também buscar por uma melhor Qualidade de vida.

É importante que os profissionais de saúde acolham adeQuadamente as mulheres climatéricas, permitindo Que exponham as suas dúvidas e receios. Além do apoio emocional e respeito, estas demandam respeito e uma assistência ajustada a suas necessidades, evitando-se intervenções desnecessárias. Não se Quer aQui desconsiderar os efeitos da terapia hormonal no alívio da sintomatologia climatérica e prevenção da osteoporose, mas lembrar a importância de outras intervenções igualmente importantes nessa fase.

As abordagens fragmentadas e reducionistas do tipo "consulta/ solicitação de exames/prescrição", nada mais Que reforçam no imaginário feminino a percepção da menopausa como um símbolo do envelhecimento e de decrepitude existencial, aumentando o sofrimento da mulher. Nesse contexto, as abordagens de caráter multidisciplinar e interdisciplinar ganham particular destąue nessa fase, por permitirem acolher um maior número de mulheres, além de favorecerem o intercâmbio de saberes e habilidades, com vistas a promover mais saúde e Qualidade de vida a essa parcela crescente da população através de um cuidado mais integral e individualizado, considerando a multiplicidades de fatores envolvidos no climatério. 


\section{REFERÊNCIAS}

I. Veloz MCT, Nascimento-Schulze CM, Camargo BV. Representações sociais do envelhecimento. Psicol Reflex Crit 1999; 12(2): 479-501.

2. Costa MFFL, Guerra HL, Barreto AS, Guimarães RM. Diagnóstico da situação de saúde da população idosa brasileira: um estudo da mortalidade e das internações hospitalares públicas. Inf Epidemiol SUS 2000; 9(I): 23-4I.

3. Ramos LR, Rosa TE, Oliveira ZM, Medina MCG, Santos FRG Perfil do idoso em área metropolitana na região sudeste do Brasil: resultados de ineuérito domiciliar. Rev Saúde Pública 2007; 27(2): 87-94.

4. Uchôa E. Contribuições da antropologia para uma abordagem das Questões relativas à saúde do idoso. Cad Saúde Pública 2003; 19(3): 849-53

5. De Lorenzi DRS, Baracat EC. Climatério e Qualidade de vida. Femina 2005; 33(12): 903-9.

6. Utian WH. Menopause, a modern perspective from a controversial history. Maturitas 1993; 26(2): 73-82.

7. Pedro AO, Pinto-Neto AM, Costa-Paiva L, Osis MJ, Hardy E. Procura de serviço médico por mulheres climatéricas brasileiras. Rev Saúde Pública 2003; 36(4): 484-90

8. Rozenbaum $\mathrm{H}$. Why has menopause became a public health problem? Therapy 1998; 53(I): 49-59.

9. Zahar SEV, Aldrighi JM, Tostes MA et al. Avaliação da Qualidade de vida na menopausa. Reprod Clim 200 I; 16(3): 163-72.

10. Aldrighi IM, Aldrighi CMS, Aldrighi APS. Alterações sistêmicas do climatério. Rev Bras Med 2002; 59(4): 15-21.

11. Bossemeyer RP. Aspectos gerais do climatério. In: Fernandes CE, Melo NR, Wehba S. Climatério feminino: fisiopatologia, diagnóstico e tratamento. São Paulo: Lemos Editoria, 1999. p. 17-33.

12. De Lorenzi DRS, Danelon C, Saciloto B, Padilha Ir I. Fatores indicadores da sintomatologia climatérica. Rev Bras Ginecol Obstet 2005; 27(1): 12-9.

13. Dennerstein L, Lehert P, Guthrie J. The effects of the menopausal transition and biopsychossocial factors on well-being. Arch of Women Mental Health 2002; 5(1): 15-22.

14. Di Corrado D, Di Nuovo S, Catalano D, Souatrito R, Sciacchitano G, Trovato GM. Quality of life in menopause. Experimental Research. Clin Terap 200 I; 152(4): 235-40.

15. Hunter MS. Predictors of menopausal symptoms: psychosocial aspects. Baillieres Clin Endocrinol Metabolism 1993; 7(1):33-45.

16. Favarato MEC, Aldrighi JM, Fráguas IR et al. Sexualidade e climatério: influência de fatores biológicos, psicológicos e sócioculturais. Reprod Clim 2000; 15(4): 199-202.

17. De Lorenzi DRS, Saciloto B. Freeüência da atividade sexual em mulheres menopausadas. Rev Assoc Med Bras 2006; 52(4): 256-60.

18. De Lorenzi DRS, Baracat EC, Saciloto B, Padilha Ir. I. Fatores Associados à Qualidade de vida na pós-menopausa. Rev Assoc Med Bras 2006; 52(5): 312-7.

19. Souza LS, Aldrighi JM. Sono e climatério. Reprod Clim 200 I; 16:20-5.

20. De Lorenzi DRS, Basso El, Fagundes PO, Saciloto B. Prevalência de sobrepeso e obesidade no climatério. Rev Bras Ginecol Obstet 2005; 27(8): 479-84.
21. Binfa L, Blümel M. Obesidad, estrógenos y salud de la mujer. Rev Chil Obstet Ginecol 2001; 66(4): 340-46.

22. Lindsay R. What have we learned from clinical studies? Fractures and the interactions of bone mass and remodeling. Osteoporos Int 2003; 14 (Suppl 5): S8-11.

23. Bassan R. Alterações cardiovasculares e cardiomorbidade da menopausa. Efeitos da terapia de reposição hormonal. Are Bras Cardiol 1999;72(1): 85-98.

24. Writing Group for the Women's Health Initiative Investigators. Risks and benefits of estrogen plus progestin in healthy postmenopausal women: principal results from Women's Health Initiative randomized controlled trial. JAMA 2002; 288(3): 321-33.

25. Sociedade Brasileira de Climatério (SOBRAC). Consenso brasileiro multidisciplinar de assistãncia à mulher climatérica. São Paulo: SOBRAC; 2003

26. Mendelsohn ME; Karas RH. HRT and the young at heart. N Engl I Med 2007; 356(25): 2639-4I.

27. Testa MA, Simonson DC. Assessment of Quality of life outcomes. N Engl I Med 1996; 334(I3): 835-40.

28. Groeneveld FPMI, Bareman FR, Barentsen R et al. Relationships between attitude towards menopause, well-being and medical attention among women aged 45-60 years. Maturitas 1993; 17(2): 77-88.

29. Merhy EE, Onocko R. Agir em Saúde: um desafio para o público. São Paulo: Hucitec; 1997.

30. Berni NIO, Luz MH, Kohlrausch SC. Conhecimento, percepções e assistência à saúde da mulher no climatério. Rev Bras Enferm 2007; 60(3): 299-306

31. Gonçalves R, Merighi MAB, Aldrighi JM. Reflexões sobre o climatério com enfoque no corpo, na cultura e na subjetividade. Reprod Clim 2003; I 8(2): 108- I 2.

32. Liao KL, Hunter MS. Preparation for menopause: prospective evaluation of a health education intervention for mid-aged women. Maturitas 1998; 29(3): 215-24.

33. Ueda M, Tokunaga M. Effects of exercise experienced in the life stages on climacteric symptoms for females. I Physiol Anthropol Appl Human Sci 2000; 19(4): I8I-9.

34. Nadai A, Nahas EAP, Burini RC. Aspectos metabólicos do exercício físico na menopausa. Femina 1999; 27(10): 791-3.

35. Ivarson T, Spertz AC, Hammar M. Physical exercise and vasomotor symptoms in postmenopausal women. Maturitas 1998; 29(2): 139-46

36. Corrêa PCRP. Tabagismo, hipertensão e diabetes - reflexões. Rev Bras Clín Terap 2003; 29(1): 19-24.

37. Fonseca AM, Polak JOM, Bagnoli VR. Menopausa e tabagismo. Rev Ginecol Obstet 1999; 10(1): 21-5.

38. Montilla RNG, Marucci MFN, Aldrighi JM. Avaliação do estado nutricional e do consumo alimentar de mulheres no climatério. Rev Assoc Med Bras 2003; 49(1): 91-5.

39. Lopes CG. Integralidade na saúde da mulher: a Questão do climatério [dissertação]. Rio de laneiro (RI): Escola Nacional de Saúde Pública Sérgio Arouca, Fundação Osvaldo Cruz; 2007.

40. Mattos RA. Integralidade e a formulação de políticas específicas de saúde. In: Pinheiro R, Mattos RA, organizadores. Construção da integralidade: cotidiano, saberes e práticas em saúde. I Ia ed. 
Rio de Janeiro: ABRASCO; 2003. p. 45-59.

41. Gonçalves R, Merighi MAB. O climatério: a corporeidade como berço das experiências do vivido. Rev Bras Enferm 2005; 58(6):
692-7.

42. De Lorenzi DRS. Avaliação da Qualidade de vida no climatério. Rev Bras Ginecol Obstet 2008; 30(3): 103-6. 